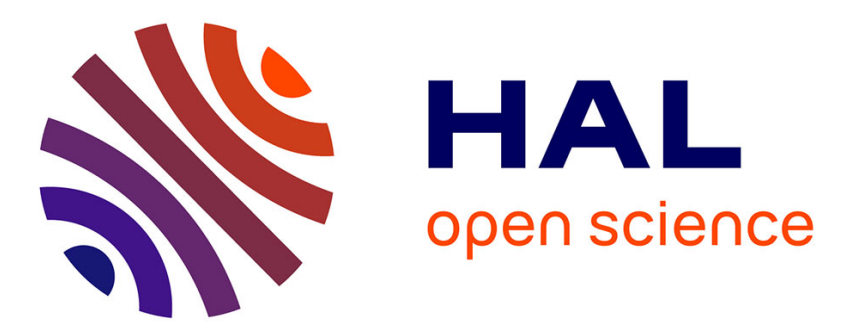

\title{
A closed-form solution for the internal stresses in thick composite cylinders induced by cyclical environmental conditions
}

Frédéric Jacquemin, Alain Vautrin

\section{- To cite this version:}

Frédéric Jacquemin, Alain Vautrin. A closed-form solution for the internal stresses in thick composite cylinders induced by cyclical environmental conditions. Composite Structures, 2002, 58, pp.1-9. 10.1016/S0263-8223(02)00051-X . hal-01004677

\section{HAL Id: hal-01004677 \\ https://hal.science/hal-01004677}

Submitted on 21 Nov 2017

HAL is a multi-disciplinary open access archive for the deposit and dissemination of scientific research documents, whether they are published or not. The documents may come from teaching and research institutions in France or abroad, or from public or private research centers.
L'archive ouverte pluridisciplinaire HAL, est destinée au dépôt et à la diffusion de documents scientifiques de niveau recherche, publiés ou non, émanant des établissements d'enseignement et de recherche français ou étrangers, des laboratoires publics ou privés. 


\title{
A closed-form solution for the internal stresses in thick composite cylinders induced by cyclical environmental conditions
}

\author{
F. Jacquemin, A Vautrin * \\ Mechanical and Materials Engineering Department, École Nationale Supérieure des Mines de Saint-Étienne, 158, cours Fauriel, \\ 42023 Saint-Étienne cedex 2, France
}

The paper presents a new semi-analytical model to assess the internal stress field in cylindrical pipes due to cyclical temperature and humidity conditions. The solution applies to both thin or thick laminated pipes composed of orthotropic plies following Fick's diffusion laws. The only restriction put on those diffusion laws is that the Arrhénius activation energy should be ply independent. Firstly, the resulting time and space dependent moisture concentration field within the interior of the pipe wall is calculated. It is the sum of the permanent solution, due to the mean relative humidity conditions imposed on the two lateral surfaces of the pipe, and a cyclical vanishing solution. Secondly, the mechanical problem is solved leading to the full stress field solution over the pipe interior and close to the lateral surfaces. The final solution can be implemented in a friendly-user software to assess the combined effects of the pipe thickness, ply stacking sequence and transient service conditions over the internal stress field.

Keywords: Hygrothermal stresses; Moisture concentration; Cyclical temperature and humidity conditions, Thick composite pipes

\section{Introduction}

The paper deals with the determination of the hygrothermal stresses in thick laminated pipes subjected to cyclical temperature and relative humidity variations on their two lateral surfaces. Those variations can differ on each boundary surface but they should be periodic functions of the same time period $\tau$.

The moisture absorption of each ply is modelled by a Fick's law whose diffusion coefficient depends on the temperature through an Arrhénius law. Since the temperature diffusion speed exceeds the moisture diffusion one by several orders, it is reasonable to assume that the thermal equilibrium is reached instantaneously. Therefore, the temperature field over the thickness of the pipe is given by the permanent solution. Furthermore, when the cyclical boundary temperatures are equal, the resulting cyclical temperature field is uniform at any time $t$. Finally, the moisture diffusion coefficients do not depend on the spatial coordinates and are time dependent periodic function of period $\tau$.

\footnotetext{
${ }^{*}$ Corresponding author.

E-mail address: vautrin@emse.fr (A. Vautrin).
}

Let us now consider a $n$-ply laminated pipe (Fig. 1). The outer and inner radii of which are denoted by $a$ and $b$ respectively. Any ply $i$ is a cylinder whose inner and outer radii are $r_{i}$ and $r_{i+1}$. The moisture concentration field in ply $i$ is solution of the system (1) and (2) below:

$$
\begin{gathered}
\frac{\partial c_{i}}{\partial t}=D_{i}(t)\left[\frac{\partial^{2} c_{i}}{\partial r^{2}}+\frac{1}{r} \frac{\partial c_{i}}{\partial r}\right] \\
a<r<b, t>0, \quad i=1 \text { to } n \\
\left\{\begin{array}{l}
c_{i}\left(r_{i}, t\right)=c_{i+1}\left(r_{i}, t\right) \\
D_{i}(t) \frac{\partial c_{i}\left(r_{i}, t\right)}{\partial r}=D_{i+1}(t) \frac{\partial c_{i+1}\left(r_{i}, t\right)}{\partial r} \\
c(a, t)=c_{a}(t) \text { and } c(b, t)=c_{b}(t) \\
c(r, 0)=0
\end{array}\right.
\end{gathered}
$$

$D_{i}(t), c_{a}(t)$ and $c_{b}(t)$ are respectively the time dependent moisture diffusion coefficient of ply $i$ and the boundary periodic concentrations at $r=a$ and $r=b$. All those time periodic functions have the same period $\tau$.

The method used to solve this problem relies on the existence of two solutions acting over two different regions of the pipe wall: a transient solution within the pipe interior and a fluctuating solution in regions very close to the two lateral surfaces subjected to the periodic boundary conditions [1]. When the number of cycles is increasing a lot, it is shown that the transient evolution 


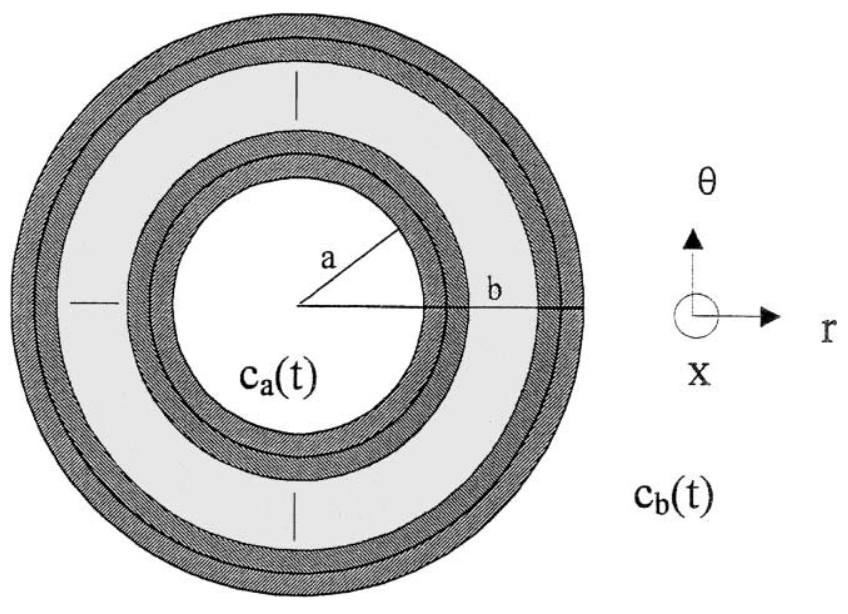

Fig. 1. Laminated hollow cylinder.

approaches a permanent state and that the fluctuating function becomes a time periodic function of period $\tau$. The regions where the fluctuating solution holds are determined by their thickness $e_{0}$. $e_{0}$ depends on the moisture diffusion coefficient, the temperature and the period of the cycles. The transient solution is obtained by an approach based on a direct analytical method and the fluctuating one is determined by using a finite difference scheme $[2,3]$. The existence of the analytical solution is based on the assumption that the activation energy of the moisture diffusion is ply independent $(3)[2,3]$.

$$
\left\{\begin{array}{l}
D_{i}(t)=A_{i} \exp \left(\frac{B_{i}}{T(t)}\right) \\
D_{i+1}(t)=A_{i+1} \exp \left(\frac{B_{i+1}}{T(t)}\right) \\
B_{i}=B_{i+1}
\end{array}\right.
$$

The transient moisture concentration field at cycle $N-k$ for ply $i$ is [2,3]:

$$
\begin{aligned}
c_{i}(\bar{r}, N-k)= & c_{\infty}+\sum_{m=1}^{\infty} \frac{2 \omega_{m}}{\Delta_{u}^{\prime}\left(\omega_{m}\right)}\left[\sum_{n=0}^{k-1} \exp \left(-\omega_{m}^{2}(N-n)\right)\right] \\
& \times\left\{A_{i} J_{0}\left(\beta_{i} \omega_{m} \bar{r}\right)+B_{i} Y_{0}\left(\beta_{i} \omega_{m} \bar{r}\right)\right\}
\end{aligned}
$$

with

$c_{\infty}=\frac{1}{\Delta_{s}}\left(A_{i}^{\prime}+B_{i}^{\prime} \ln \bar{r}\right)$

The transient moisture concentration (4) at cycle $N-k$ is the sum of the permanent solution $c_{\infty}$ reached after $N$ cycles and the transient solution written in form of series. $J_{0}$ and $Y_{0}$ are Bessel functions of zero order and $\bar{r}$ is the dimensionless radius defined by the relation $\bar{r}=r / b$. All the terms determining the transient moisture concentration are detailed in Refs. [2,3].

The concentration (4) obviously does not satisfy the periodic boundary conditions. In fact, as previously pointed out, the transient solution is strictly valid within the pipe interior at a distance $e_{0}$ from the lateral sur- faces. This distance $e_{0}$ has been estimated by Verchery in the case of a laminated plate [4]:

$e_{0}=2 \sqrt{\pi \int_{0}^{\tau} D(t) \mathrm{d} t}$

In the regions where the fluctuating solution prevails, that is in the cylinders of thickness $e_{0}$, the concentration fields are estimated by a finite difference computation as said before.

We will discuss later two examples showing the effect of a cyclical hygrothermal loading on the moisture concentration within thick laminated pipes whose plies have either identical (so-called homogeneous case) or different moisture diffusion coefficients (heterogeneous case).

\section{Mechanical problem}

At the initial time, let us assume that the pipe is stress free. Therefore, the hygrothermoelastic orthotropic behaviour writes as follow in (6) and (7), where $\alpha, \beta$ and $\mathbf{L}$ are respectively the in-plane tensors of thermal and hygroscopic expansion coefficients and moduli. Those tensors are assumed to be material constants.

$$
\begin{aligned}
\left\{\begin{array}{l}
\sigma_{x x} \\
\sigma_{\theta \theta} \\
\sigma_{r r} \\
\tau_{x \theta}
\end{array}\right\}= & {\left[\begin{array}{llll}
L_{x x} & L_{x \theta} & L_{x r} & L_{x s} \\
L_{x \theta} & L_{\theta \theta} & L_{r \theta} & L_{s \theta} \\
L_{x r} & L_{r \theta} & L_{r r} & L_{r s} \\
L_{x s} & L_{s \theta} & L_{r s} & L_{s s}
\end{array}\right] } \\
& \times\left\{\begin{array}{l}
\varepsilon_{x x}-\alpha_{x x}\left(T-T_{0}\right)-\beta_{x x}\left(m-m_{0}\right) \\
\varepsilon_{\theta \theta}-\alpha_{\theta \theta}\left(T-T_{0}\right)-\beta_{\theta \theta}\left(m-m_{0}\right) \\
\varepsilon_{r r}-\alpha_{r r}\left(T-T_{0}\right)-\beta_{r r}\left(m-m_{0}\right) \\
\gamma_{x \theta}-\alpha_{x \theta}\left(T-T_{0}\right)-\beta_{x \theta}\left(m-m_{0}\right)
\end{array}\right\} \\
\left\{\begin{array}{l}
\tau_{r \theta} \\
\tau_{x r}
\end{array}\right\}= & {\left[\begin{array}{ll}
L_{r \theta r \theta} & L_{r \theta x r} \\
L_{x r r \theta} & L_{x r r r}
\end{array}\right]\left\{\begin{array}{l}
\gamma_{r \theta} \\
\gamma_{x r}
\end{array}\right\} }
\end{aligned}
$$

where $m=m_{\text {water }} / m_{\text {dry }}=c / \rho_{s}$. To solve the hygrothermomechanical problem, it is necessary to express the strains versus the displacements along with the compatibility and equilibrium equations.

Introducing a characteristic length $L_{0}$, we introduce the following dimensionless variables:

$\bar{\sigma}=\sigma / L_{0}, \quad \mathbf{L}=\mathbf{L} / L_{0}, \quad(\bar{w}, \bar{u}, \bar{v})=(w, u, v) / b$.

Displacements with respect to $x$ and $\theta$, respectively $\bar{u}(\bar{x}, \bar{r})$ and $\bar{v}(\bar{x}, \bar{r})$ are then deduced:

$$
\left\{\begin{array}{l}
\bar{u}(\bar{x}, \bar{r})=-\bar{S}_{r \theta x x} \frac{R_{3}}{\bar{r}}+\bar{S}_{x r x x} R_{4} \ln \bar{r}+R_{1} \bar{x}+R_{5} \\
\bar{v}(\bar{x}, \bar{r})=R_{2} \bar{x} r-\frac{\bar{S}_{r \theta r \theta}}{2} \frac{R_{3}}{\bar{r}}-\bar{S}_{r \theta x x} R_{4}+R_{6} \bar{r} \\
R_{5}, R_{6} \text { are constants and } \mathbf{S}=\mathbf{L}^{-1}
\end{array}\right.
$$

It is worth noticing that the displacements $\bar{u}(\bar{x}, \bar{r})$ and $\bar{v}(\bar{x}, \bar{r})$ do not depend at all upon the hygrothermal field 
and therefore are valid over any moisture concentration regions. Finally, to obtain the through-thickness or radial component of the displacement $\bar{w}$, we shall consider in the following firstly the analytical transient concentration, acting within the pipe interior, and secondly the fluctuating concentrations, close to the lateral surfaces.

\subsection{Radial displacement due to the transient moisture concentration}

The radial component of the displacement field $\bar{\omega}$ satisfies the following equation:

$\bar{r}^{2} \frac{\partial^{2} \bar{w}}{\partial \bar{r}^{2}}+\bar{r} \frac{\partial \bar{w}}{\partial \bar{r}}-\frac{\bar{L}_{\theta \theta}}{\bar{L}_{r r}} \bar{w}=\frac{\bar{r}\left[\left(\bar{L}_{x \theta}-\bar{L}_{x r}\right) R_{1}+\left(\bar{L}_{s \theta}-2 \bar{L}_{r s}\right) R_{2} \bar{r}\right]}{\bar{L}_{r r}}$
$+\frac{\bar{r}\left[\left(I_{1}-I_{2}\right)\left(T-T_{0}\right)+\left(K_{1}-K_{2}\right)\left(m-m_{0}\right)+K_{1} \bar{r} \frac{\partial m}{\partial \bar{r}}\right]}{\bar{L}_{r r}}$

with,

$$
\begin{aligned}
& I_{1}=\bar{L}_{x r} \alpha_{x x}+\bar{L}_{r \theta} \alpha_{\theta \theta}+\bar{L}_{r r} \alpha_{r r}+\bar{L}_{r s} \alpha_{x \theta}, \\
& I_{2}=\bar{L}_{x \theta} \alpha_{x x}+\bar{L}_{\theta \theta} \alpha_{\theta \theta}+\bar{L}_{r \theta} \alpha_{r r}+\bar{L}_{s \theta} \alpha_{x \theta}, \\
& K_{1}=\bar{L}_{x r} \beta_{x x}+\bar{L}_{r \theta} \beta_{\theta \theta}+\bar{L}_{r r} \beta_{r r}+\bar{L}_{r s} \beta_{x \theta}, \\
& K_{2}=\bar{L}_{x \theta} \beta_{x x}+\bar{L}_{\theta \theta} \beta_{\theta \theta}+\bar{L}_{r \theta} \beta_{r r}+\bar{L}_{s \theta} \beta_{x \theta}
\end{aligned}
$$

It is shown that the general solution of Eq. (9) writes as the sum of a solution of the homogeneous equation and of a particular solution. Considering the transient moisture concentration (4), we obtain the radial component of the displacement field within the pipe:

$$
\begin{aligned}
\bar{w}= & R_{7} \bar{r} \sqrt{\bar{L}_{\theta \theta} / \bar{L}_{r r}}+R_{8} \bar{r}^{-} \sqrt{\bar{L}_{\theta \theta} \bar{L}_{r r}}+\frac{\left(\bar{L}_{x \theta}-\bar{L}_{x r}\right) R_{1} \bar{r}}{\bar{L}_{r r}\left(1-\frac{\bar{L}_{\theta \theta}}{\bar{L}_{r r}}\right)} \\
+ & \frac{\left(I_{1}-I_{2}\right)\left(T-T_{0}\right) \bar{r}}{\bar{L}_{r r}\left(1-\frac{\bar{L}_{\theta \theta}}{\bar{L}_{r r}}\right)}+\frac{\left(\bar{L}_{s \theta}-2 \bar{L}_{r s}\right) R_{2} \bar{r}^{2}}{\bar{L}_{r r}\left(4-\frac{\bar{L}_{\theta \theta}}{\bar{L}_{r r}}\right)}-\frac{\left(K_{1}-K_{2}\right) m_{0} \bar{r}}{\bar{L}_{r r}\left(1-\frac{\bar{L}_{\theta \theta}}{\bar{L}_{r r}}\right)} \\
+ & \frac{\left(K_{1}-K_{2}\right) A_{i}^{\prime} \bar{r}}{\rho_{s} \bar{L}_{r r}\left(1-\frac{\bar{L}_{\theta \theta}}{\bar{L}_{r r}}\right) \Delta_{s}}+\frac{B_{i}^{\prime}}{\rho_{s} \Delta_{s}}\left[\frac{\left(K_{1}-K_{2}\right) \bar{r} \ln \bar{r}+K_{1} \bar{r}}{\bar{L}_{r r}\left(1-\frac{\bar{L}_{\theta \theta}}{\bar{L}_{r r}}\right)}\right. \\
& \left.-\frac{2\left(K_{1}-K_{2}\right) \bar{r}}{\bar{L}_{\theta \theta}}\right)^{2} \\
+ & \frac{1}{\rho_{s}} \sum_{m=1}^{\infty} \frac{2 \omega_{m}}{\bar{L}_{r r}^{\prime}\left(\omega_{m}\right)}\left[\sum_{n=1}^{k-1} \exp \left(-\omega_{m}^{2}(N-n)\right)\right] \\
& \times\left[A _ { i } \left(\frac{\left(K_{1}-K_{2}\right)}{\bar{L}_{r r}} \sum_{k=0}^{\infty} \frac{(-1)^{k}\left(\frac{1}{2} \beta_{i} \omega_{m}\right)^{2 k}}{(k !)^{2}} \frac{\bar{r}^{(2 k+1)}}{\left.(2 k+1)^{2}-\frac{\bar{L}_{\theta \theta}}{\bar{L}_{r r}}\right)} .\right.\right.
\end{aligned}
$$

$$
\begin{aligned}
& \left.-\frac{K_{1}}{\bar{L}_{r r}} \sum_{k=0}^{\infty} \frac{(-1)^{k}\left(\frac{1}{2}\right)^{2 k+1}\left(\beta_{i} \omega_{m}\right)^{2 k+2}}{k !(k+1) !} \frac{\bar{r}^{(2 k+3)}}{\left.(2 k+3)^{2}-\frac{\bar{L}_{\theta \theta}}{\bar{L}_{r r}}\right)}\right\} \\
& +\frac{B_{i}}{\pi}\left\{\frac{2\left(K_{1}-K_{2}\right)}{\bar{L}_{r r}} \sum_{k=0}^{\infty} \frac{(-1)^{k}\left(\frac{1}{2} \beta_{i} \omega_{m}\right)^{2 k}}{(k !)^{2}}\right. \\
& \times\left[\ln \left(\frac{1}{2} \beta_{i} \omega_{m} \bar{r}\right)-\frac{2(2 k+1)}{\left.(2 k+1)^{2}-\frac{\bar{L}_{\theta \theta}}{\bar{L}_{r r}}\right)}-\psi(k+1)\right. \\
& \times \\
& \times\left[\frac{\bar{r}^{(2 k+1)}}{\left.(2 k+1)^{2}-\frac{\bar{L}_{\theta \theta}}{\bar{L}_{r r}}\right)}-\frac{K_{1}}{\bar{L}_{r r}}\left[\sum_{k=0}^{\infty} \frac{(-1)^{k}\left(\frac{1}{2}\right)^{1+2 k}\left(\beta_{i} \omega_{m}\right)^{2+2 k}}{k !(k+1) !}\right.\right. \\
& \times \frac{\left.\left(\frac{1}{2} \beta_{i} \omega_{m} \bar{r}\right)-\psi(k+1)-\psi(k+2)\right]}{\bar{r}^{(2 k+3)}} \\
& \times\left(\frac{\left.(2 k+3)^{2}-\frac{\bar{L}_{\theta \theta}}{\bar{L}_{r r}}\right)}{(2 k+3) \bar{r}^{(2 k+3)}} \sum_{k=0}^{\infty} \frac{(-1)^{k}\left(\frac{1}{2}\right)^{1+2 k}\left(\beta_{i} \omega_{m}\right)^{2+2 k}}{k !(k+1) !}\right. \\
& \left.(2 k+3)^{2}-\frac{\bar{L}_{\theta \theta}}{\bar{L}_{r r}}\right)^{2}
\end{aligned}
$$

with, $\psi(k+1)=\sum_{m=1}^{k} 1 / m+\gamma, \gamma=-0.5772157 \ldots$ (Euler's constant), for, $\bar{L}_{\theta \theta} / \bar{L}_{r r} \neq(2 k+1)^{2}$, and, $\bar{L}_{\theta \theta} / \bar{L}_{r r} \neq(2 k+3)^{2}$.

\subsection{Radial displacement due to the fluctuating moisture concentration}

The fluctuating concentration is determined by using a finite difference scheme. In order to propose a closed form solution of the displacement, we subdivide the regions close to the two lateral surfaces and assume that on each subdivision a parabolic moisture concentration holds (10):

$c_{i}=A_{i}^{\prime} \bar{r}^{2}+B_{i}^{\prime} \bar{r}+C_{i}^{\prime}$

We finally obtain the radial component of the displacement field (9) for each subdivision of the finite difference calculation:

$$
\begin{gathered}
\bar{w}=R_{7} \bar{r}^{\sqrt{\bar{L}_{\theta \theta} / \bar{L}_{r r}}}+R_{8} \bar{r}^{-} \sqrt{\bar{L}_{\theta \theta} / \bar{L}_{r r}}+\frac{\left(\bar{L}_{x \theta}-\bar{L}_{x r}\right) R_{1} \bar{r}}{\bar{L}_{r r}\left(1-\frac{\bar{L}_{\theta \theta}}{\bar{L}_{r r}}\right)} \\
+\frac{\left(I_{1}-I_{2}\right)\left(T-T_{0}\right) \bar{r}}{\bar{L}_{r r}\left(1-\frac{\bar{L}_{\theta \theta}}{\bar{L}_{r r}}\right)}+\frac{\left(\bar{L}_{s \theta}-2 \bar{L}_{r s}\right) R_{2} \bar{r}^{2}}{\bar{L}_{r r}\left(4-\frac{\bar{L}_{\theta \theta}}{\bar{L}_{r r}}\right)}
\end{gathered}
$$




$$
\begin{aligned}
- & \frac{\left(K_{1}-K_{2}\right) m_{0} \bar{r}}{\bar{L}_{r r}\left(1-\frac{\bar{L}_{\theta \theta}}{\bar{L}_{r r}}\right)}+\frac{\left(K_{1}-K_{2}\right)}{\rho_{i} \bar{L}_{r r}} \\
\times & {\left[\frac{C_{i}^{\prime} \bar{r}}{\left.1-\frac{\bar{L}_{\theta \theta}}{\bar{L}_{r r}}\right)}+\frac{B_{i}^{\prime} \bar{r}^{2}}{\left(4-\frac{\bar{L}_{\theta \theta}}{\bar{L}_{r r}}\right)}+\frac{A_{i}^{\prime} \bar{r}^{3}}{\left(9-\frac{\bar{L}_{\theta \theta}}{\bar{L}_{r r}}\right)}\right] } \\
+ & \frac{K_{1}}{\rho_{i} \bar{L}_{r r}}\left[\frac{B_{i}^{\prime} \bar{r}^{2}}{\left.4-\frac{\bar{L}_{\theta \theta}}{\bar{L}_{r r}}\right)}+\frac{2 A_{i}^{\prime} \bar{r}^{3}}{\left.\left(9-\frac{\bar{L}_{\theta \theta}}{\bar{L}_{r r}}\right)\right]}\right.
\end{aligned}
$$

\subsection{Determination of the eight constants per ply}

Finally, the displacement field within each ply depends on eight constants to be determined: $R_{i}$ for $i=$ $1, \ldots, 8$. These eight constants result from the following conditions:

- rigid body motions restrained;

- displacement continuity at the interplies;

- global force balance of the cylinder;

- transverse shear stress continuity at the interplies;

- normal stress continuity at the interplies and the nullity of it on the two lateral surfaces.

\section{Examples}

Let us here consider a thick angle-ply pipe made up of five orhtotropic unidirictional T300/5208 carbon fibre/ polymer matrix plies of equal thickness. The inner and outer pipe radii are respectively $a=10 \mathrm{~mm}$ and $b=30$ $\mathrm{mm}$. Two lamination sequences, respectively referred to as homogeneous and heteregeneous case, will be studied:

1. $[+55,-55,+55,-55,+55]$, the fibre angle is defined versus the longitudinal axis.

2. [resin, $-55,+55,-55,+55]$, the first ply being made of pure N 5208 resin (liner).

The same environmental time-dependent boundary conditions are applied at $r=a$ and $r=b$. The period of the temperature and relative humidity cycles is 4 weeks as shown in Figs. 2 and 3. To point out the efficiency of the method, the moisture concentration and internal stresses have been computed at five different points of every cycle. As a matter of fact, these particular points have been selected because they characterise important changes of the relative humidity or temperature.

It is reasonable to first assume that the activation energy of the plies is equal to the activation energy of the pure resin N 5208 [5] since the diffusion process basically

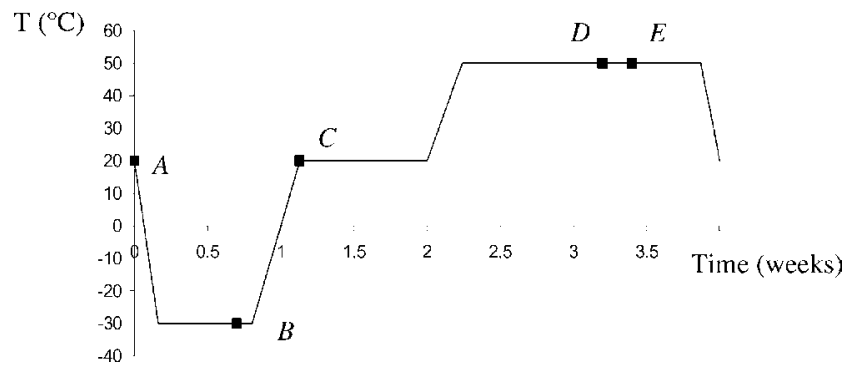

Fig. 2. Temperature cycle.

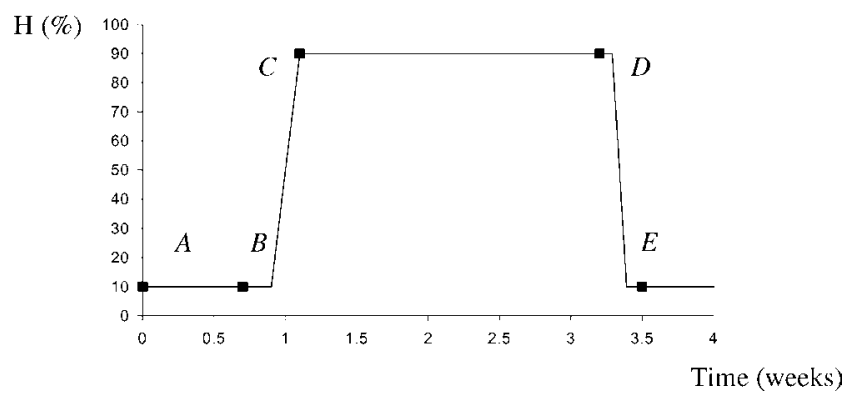

Fig. 3. Relative humidity cycle.

relies on the resin properties (Tables 1 and 2). The composite and pure resin mechanical properties in the fibre reference frame $(1,2,3)[6]$ are presented in Table 3 .

A first estimate of the hygrothermal stress state can be drawn from the Tsai-Wu strength criterion. The ply strengths utilised to run the different numerical simulations are reported in Table 4 with usual notations. In this first approach, it has been assumed that those strengths do not depend on moisture content and temperature. As a matter of fact, there will be no particular difficulty to take into account any experimental variation of those material properties versus the environmental parameters. Finally, the strength factor $R$ is classically derived from the Tsai-Wu criterion, therefore first-ply failure could occur when $R$ is approaching 1 .

Table 1

Hygroscopic properties of T300/5208 UD ply

\begin{tabular}{ll} 
Diffusion coefficient $\left(\mathrm{mm}^{2} / \mathrm{s}\right)$ & $D(t)=0.57 \exp (-5116 / T(t))$ \\
& $T(\mathrm{~K})$ \\
Moisture concentration $\left(\mathrm{kg} / \mathrm{m}^{3}\right)$ & $C=0.2385 H$ \\
& $H(\%)$ \\
\hline
\end{tabular}

Table 2

Hygroscopic properties of N 5208 neat resin

\begin{tabular}{ll} 
Diffusion coefficient $\left(\mathrm{mm}^{2} / \mathrm{s}\right)$ & $D(t)=2.8 \exp (-5116 / T(t))$ \\
& $T(\mathrm{~K})$ \\
Moisture concentration $\left(\mathrm{kg} / \mathrm{m}^{3}\right)$ & $C=0.708 H$ \\
& $H(\%)$ \\
\hline
\end{tabular}


Table 3

Mechanical properties in the UD orthotropic frame $(1,2,3)$

\begin{tabular}{llllllllll}
\hline Material & $E_{1}(\mathrm{GPa})$ & $E_{2}, E_{3}(\mathrm{GPa})$ & $v_{12}, v_{13}$ & $v_{23}$ & $G_{12}(\mathrm{GPa})$ & $\alpha_{1}\left(\mathrm{~K}^{-1}\right)$ & $\alpha_{2}, \alpha_{3}\left(\mathrm{~K}^{-1}\right)$ & $\beta_{1}$ & $\beta_{2}, \beta_{3}$ \\
\hline T300/5208 & 181 & 10.3 & 0.28 & 0.43 & 7.17 & $0.02 \times 10^{-6}$ & $22.5 \times 10^{-6}$ & 0 & 0.6 \\
N5208 & 4.5 & 4.5 & 0.4 & 0.4 & 1.6 & $11 \times 10^{-5}$ & $11 \times 10^{-5}$ & 0.6 & 0.6 \\
\hline
\end{tabular}

Table 4

T300/5208 mechanical strengths [6]

\begin{tabular}{lllll}
\hline Strengths (MPa) & $X, X^{\prime}$ & $Y, Z$ & $Y^{\prime}, Z^{\prime}$ & $S$ \\
\hline T300/5208 & 1500 & 40 & 246 & 68 \\
\hline
\end{tabular}

For the pure resin region, i.e. for the internal ply in the heterogeneous case (case 2), the von Mises yield criterion is applied. The value of the yield stress $\sigma_{0}$ is 130 $\mathrm{MPa}$ [7]. This value is assumed to be independent of both the temperature and moisture content.

\subsection{Homogeneous case (case 1)}

Since the transient moisture field within the pipe interior (Fig. 4) only results from the mean moisture concentrations at the boundary, the same moisture content is reached for $r=a$ and $r=b$ those. Furthermore, the permanent moisture content obtained after some time is shown to be constant for the same reason. Finally, the penetration lengths $e_{0}$ of the fluctuating moisture concentration are equal and both the finite difference and the analytical calculation of the transient concentration lead to the same results.

\subsubsection{Periodic-permanent solutions}

The fluctuating moisture content is prevailing in two regions, close to the lateral surfaces, whose thickness $e_{0}$ is about $1 \mathrm{~mm}$. After some time, this fluctuating solution becomes a periodic solution (Fig. 5). It can be clearly noticed in Fig. 5 that the constant permanent solution holds at a distance $e_{0}$ from the surfaces.

The permanent through-thickness normal stress $\sigma_{r r}$ (Fig. 6) is the same for both the couples (A,C) and (D,E) which correspond to identical temperatures but to different moisture contents imposed on the boundaries.

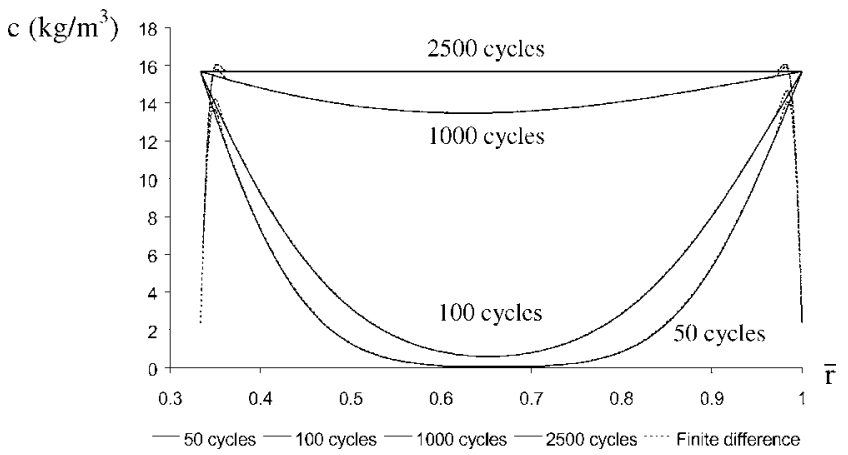

Fig. 4. Transient moisture concentration.

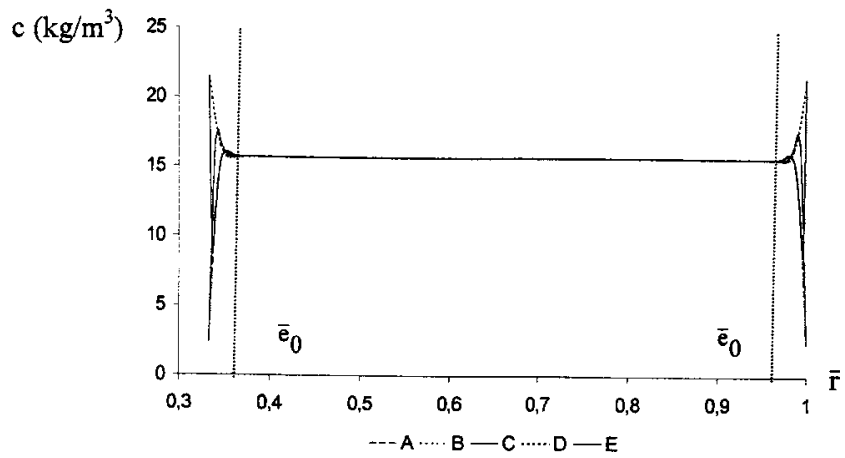

Fig. 5. Moisture concentration in the periodic-permanent state.

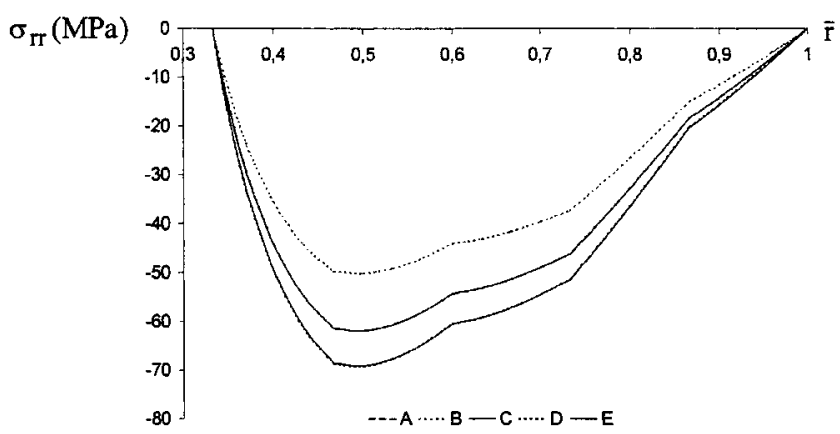

Fig. 6. Radial normal stress.

This radial stress is maximum at point $\mathrm{B}$ because of the tensile stress due to the temperature drop between $\mathrm{A}$ and B states. It is emphasised that the radial stress is primarily dependent on the temperature variation since it is equal to zero on the boundaries.

The in-plane stress $\sigma_{2}$ normal to the fibres is sensitive to the cyclical concentration gradients close to the boundaries, where the fluctuating solution prevails (Fig. 7). In these regions, the stress gradients are so high that the stress can be alternated. The hygrothermal strength factor gives some insight into the stress gradient effects especially near the external surface (Fig. 8). A sudden drop of the strength factor is detected when the normal transverse stress becomes positive (Fig. 7).

\subsubsection{Fluctuating-transient solution during cycle 50}

During cycle 50 , the moisture concentration field is obviously independent of time except in the two fluctuating concentration regions, near the lateral surfaces, whose thickness has been previously estimated by $e_{0}$ (Fig. 9). 


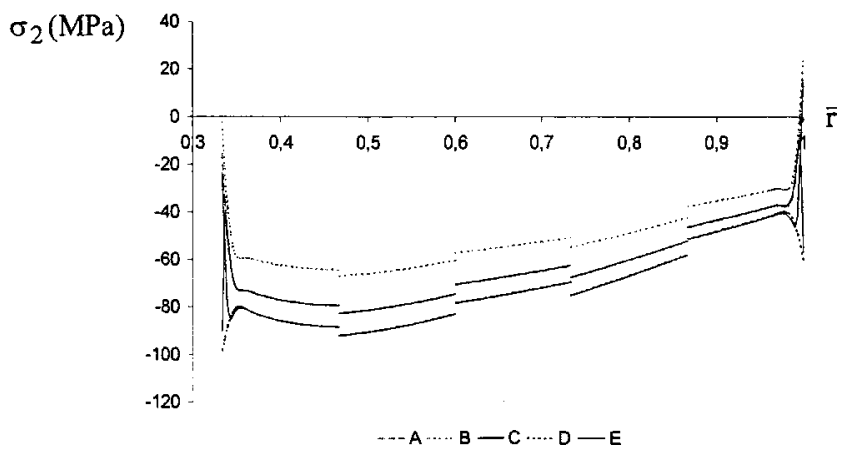

Fig. 7. In-plane stress normal to the fibres.

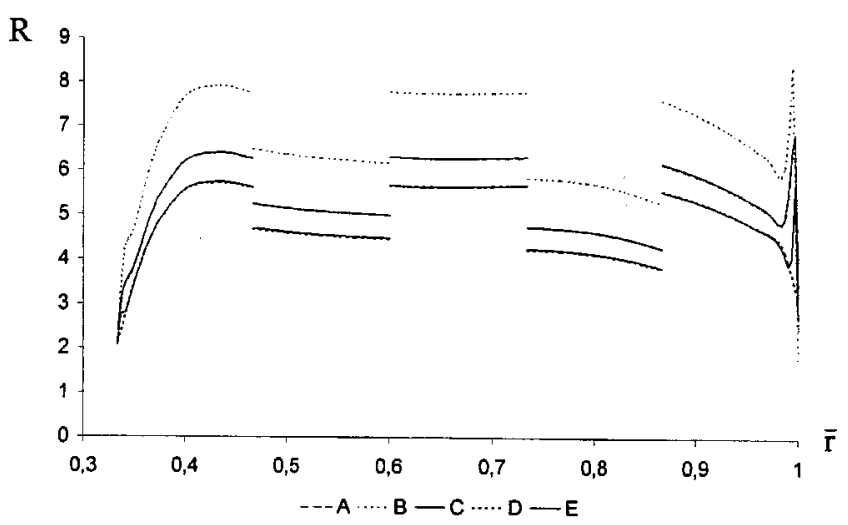

Fig. 8. Hygrothermal strength factor.

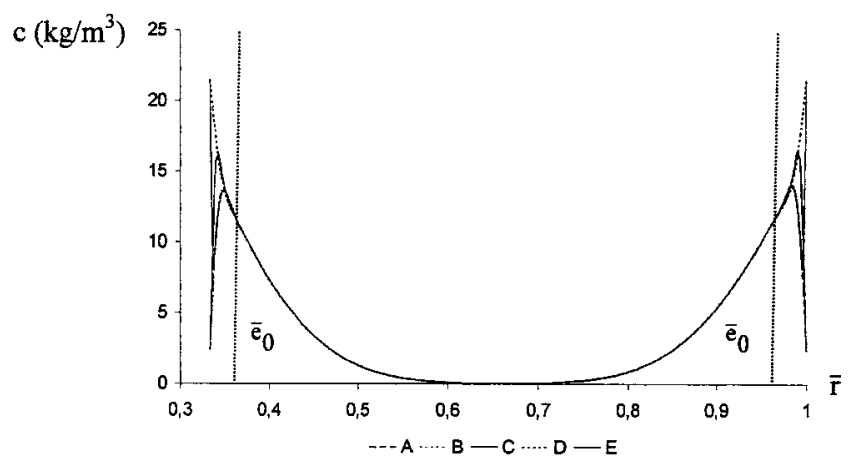

Fig. 9. Moisture concentration during cycle 50.

The couples $(\mathrm{A}, \mathrm{C})$ and $(\mathrm{D}, \mathrm{E})$ are leading to very similar through-thickness normal stress $\sigma_{r r}$ (Fig. 10). Due to the temperature change, positive values of this stress component can be reached at point B. Such values can be very critical for the durability of the pipe. Therefore, it should be strongly recommended to simulate the stress change during the first cycles, in particular when sudden drops of the temperature occur.

The in-plane normal ply stress $\sigma_{2}$ presents high gradients in regions where the fluctuating moisture solution holds (Fig. 11). The thermal effects induce tensile stresses at point B. The strength factor undergoes important gradients over the fluctuating zones and it reaches a

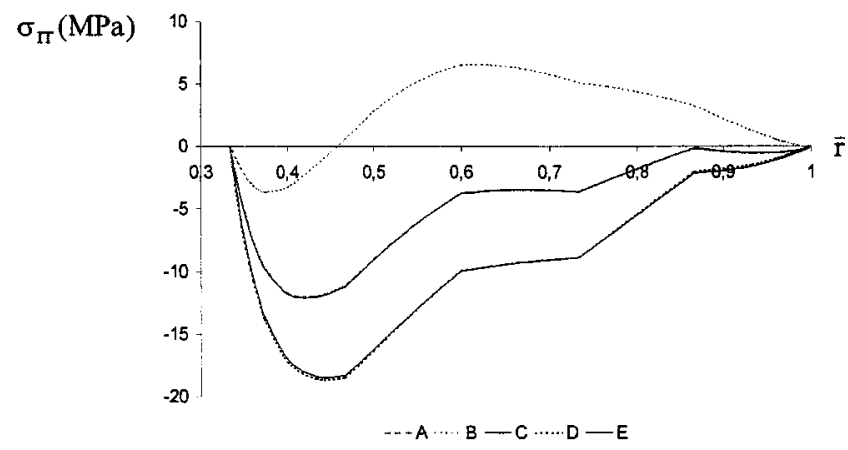

Fig. 10. Radial normal stress during cycle 50.

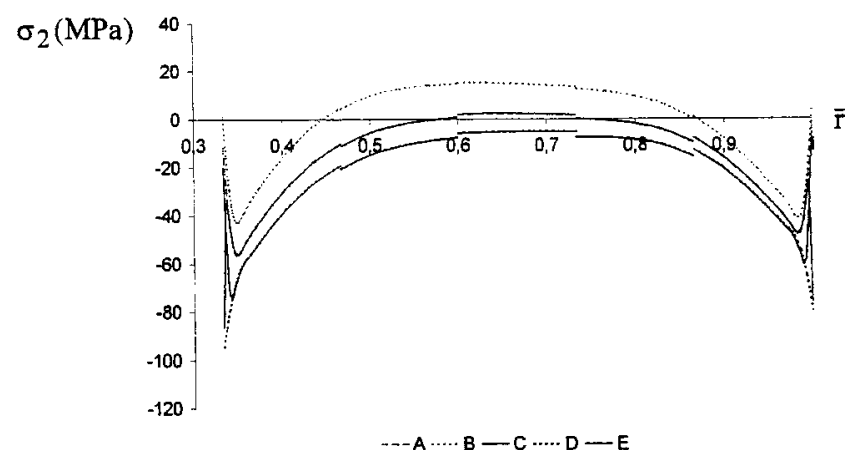

Fig. 11. In-plane stress normal to the fibres during cycle 50.

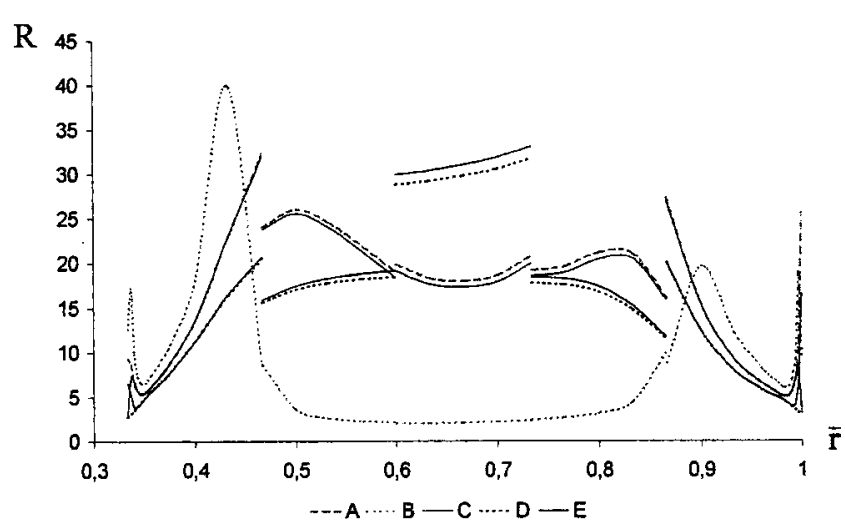

Fig. 12. Hygrothermal strength factor during cycle 50 .

minimum value of about 2 within the pipe at point $\mathrm{B}$ (Fig. 12).

\subsection{Heterogeneous case (case 2)}

The boundary conditions over the pipe walls in terms of relative humidity and temperature are still identical, however the external plies have now different hygroscopic properties (Tables 1 and 2). As a result, the boundary moisture concentrations are different and the concentration over the pure resin surface is the highest (Fig. 13). Moreover, due to the greater value of the resin 
diffusion coefficient, the extent $e_{0}$ of the fluctuating solution is also more important.

\subsubsection{Periodic-permanent solution}

The cyclical concentration is acting over two regions whose thickness is now different (Fig. 14). The thickness $e_{0}$ is more important in the resin region and reaches about $2.5 \mathrm{~mm}$. In the homogeneous case, the interlaminar normal stress $\sigma_{r r}$ in permanent state was a function of the temperature only (Fig. 6). In the present case, we note that a slight effect of a change in the moisture concentration can be detected upon the stress in the resin ply and the first composite ply (Fig. 15). Therefore, the cyclical moisture conditions, which induce more

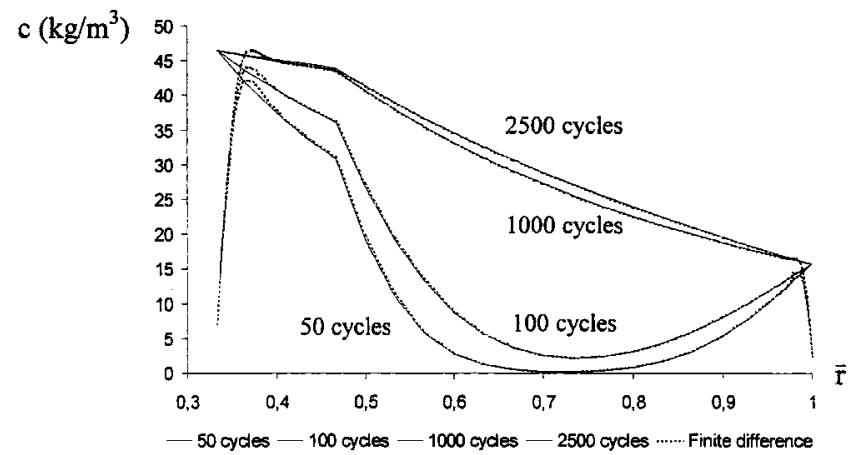

Fig. 13. Transient moisture concentration.

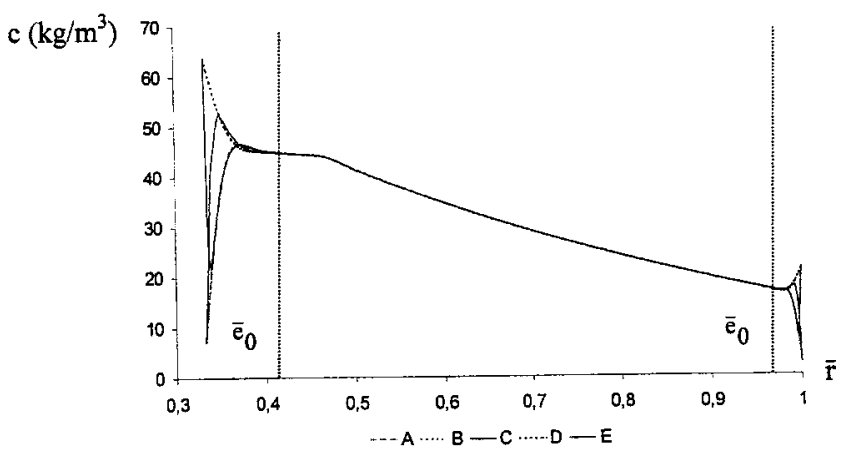

Fig. 14. Moisture concentration in the periodic permanent state.

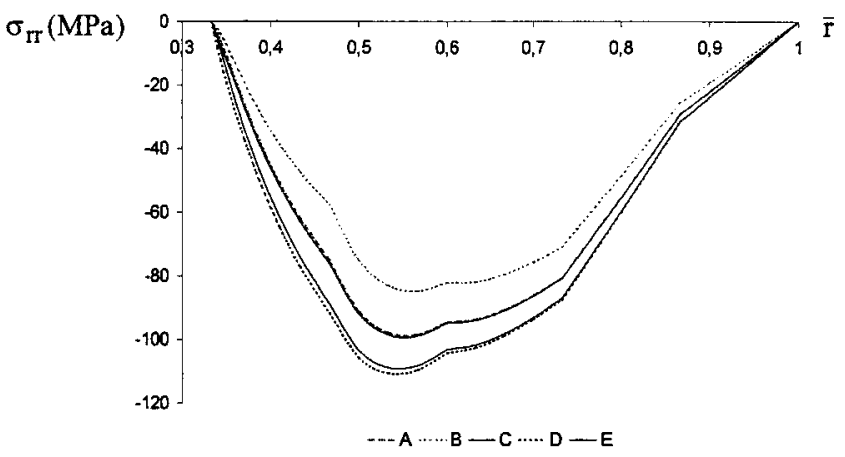

Fig. 15. Radial normal stress. important gradients over the internal resin ply, could have some significant effect on the radial stress.

In the resin region, i.e. an isotropic region, $\sigma_{2}$ is the hoop stress. In the composite region, $\sigma_{2}$ is still the inplane stress normal to the fibres (Fig. 16). It reaches quite high values and gradients, near the internal surface, which are critical for the structure. The von Mises stress reaches values which are higher than the ultimate stress (Fig. 17). Therefore, the strength factor reaches critical values, i.e. values close to 1 , on the external edge due to the in-plane stress gradients (Fig. 18).

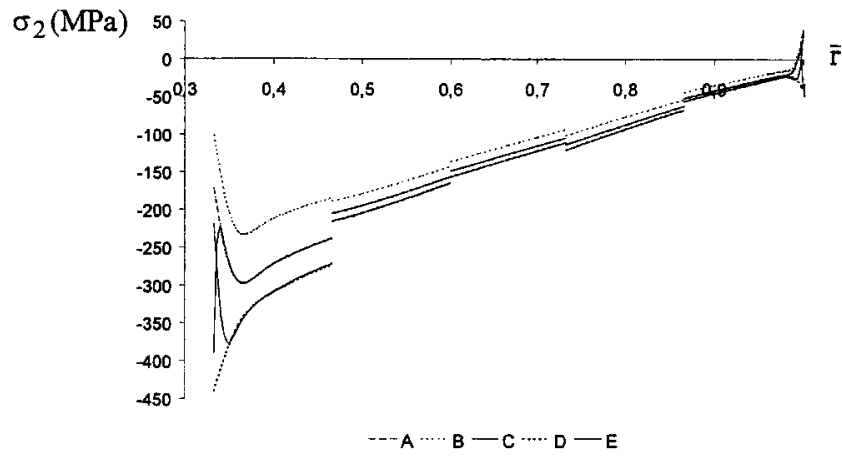

Fig. 16. In-plane stress normal to the fibres.

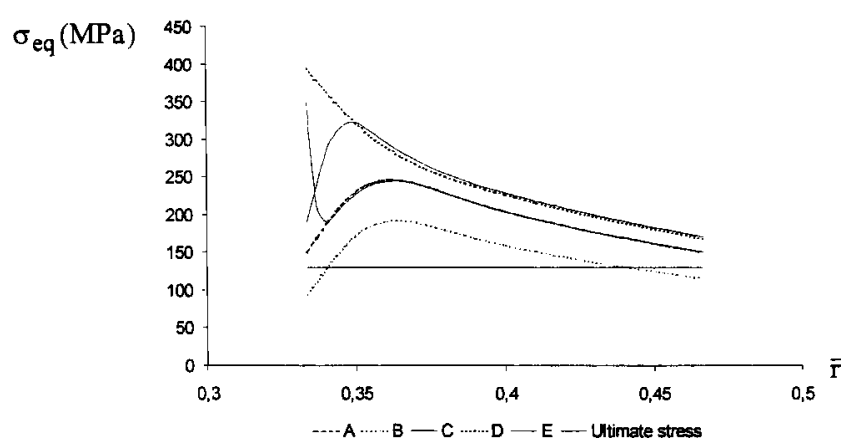

Fig. 17. von Mises equivalent stress.

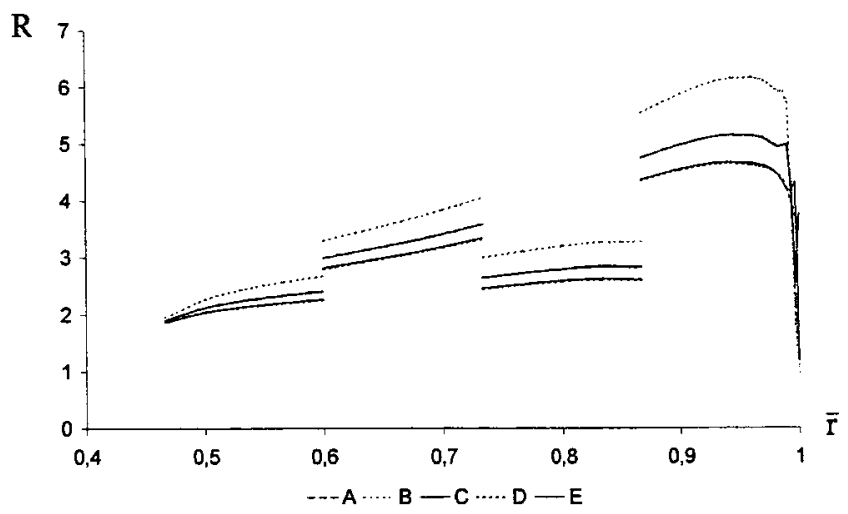

Fig. 18. Hygrothermal strength factor. 


\subsubsection{Fluctuating-transient pattern during cycle 50}

The main objective here is to emphasise how important it is to perform a stress analysis since the beginning of the cyclical process to predict ply failure. The moisture concentration during cycle 50 is plotted in Fig. 19. The interlaminar stress during cycle 50 is sensitive to both the temperature and the moisture content (Fig. 20). Indeed, those stresses are now clearly distinct for couples $(\mathrm{A}, \mathrm{C})$ and $(\mathrm{D}, \mathrm{E})$. The extend of the fluctuating concentration region and the high time dependent gradients within the pipe walls can give rise to this particular effect.

The hoop stress in the resin ply (Fig. 21) is strongly affected by the moisture concentration gradients. How-

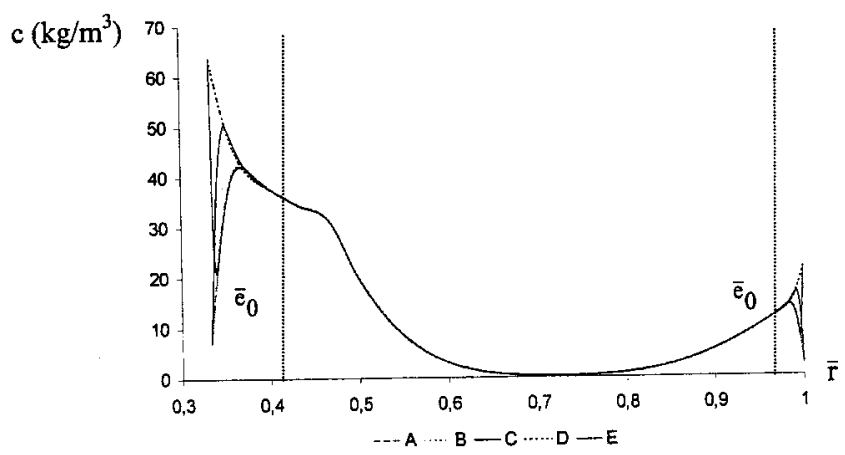

Fig. 19. Moisture concentration during cycle 50.

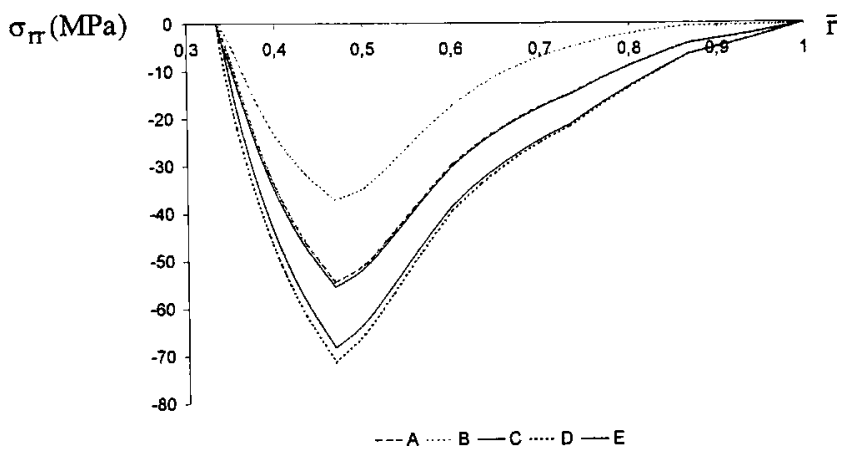

Fig. 20. Radial normal stress during cycle 50.

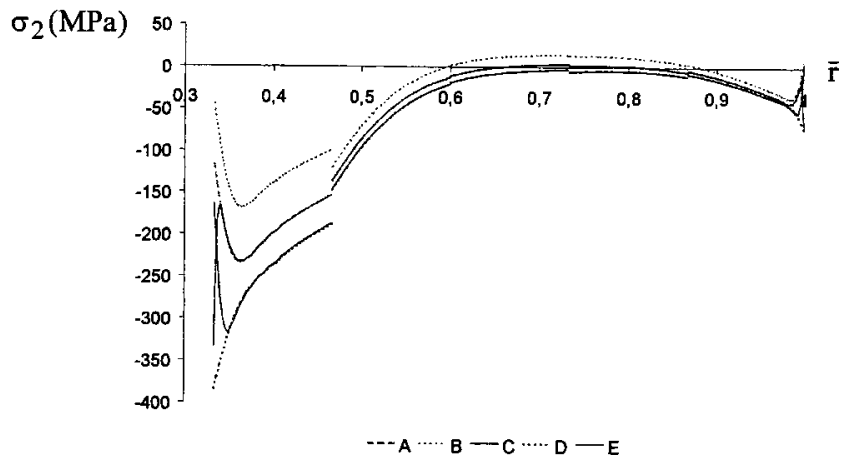

Fig. 21. In-plane stress normal to the fibres during cycle 50.

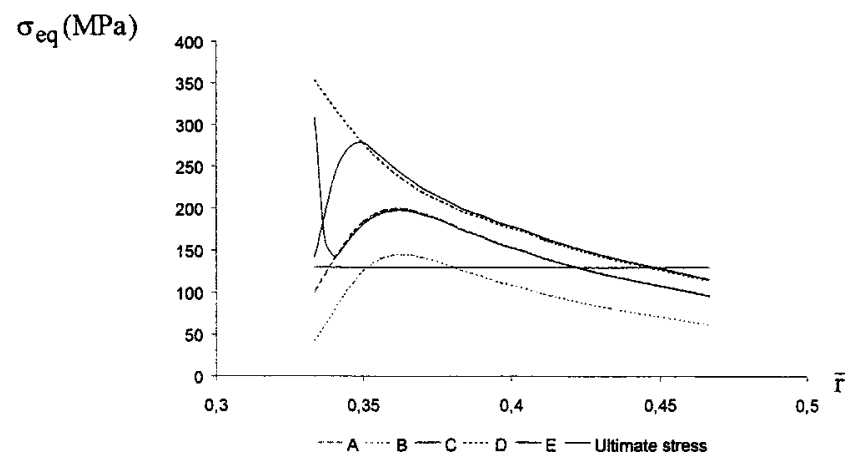

Fig. 22. von Mises equivalent stress during cycle 50

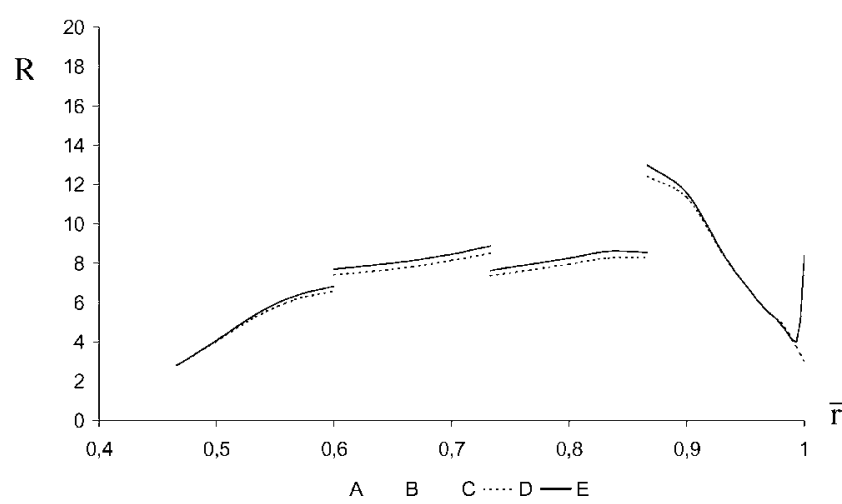

Fig. 23. Hygrothermal strength factor $R$ during cycle 50 .

ever, the interlaminar stress has a positive value within the pipe in the composite region. Thus, considering the first cycles - in the present case cycle 50 -is clearly relevant to point out the resin failure which may occur quite soon (Fig. 22). Still, the strength factor remains encouraging for the composite plies (Fig. 23).

\section{Conclusion}

The paper presents a semi-analytical method to assess the transient moisture concentration and related internal stresses for thick laminated composite pipes submitted to hygrothermal cyclical conditions on their lateral surfaces. This semi-analytical method allows to check the influence of concentration or temperature changes on the material stress state. Moreover, this approach can be applied to the heterogeneous ply arrangements, whose diffusion coefficient have the same activation energy: for instance when the resin matrix is identical from ply to ply.

The thermal effects which occur during the different cooling and heating phases of the cycle are usually quite distinct from those due to relative humidity changes. Those latter effects are fundamentally characterised by the existence of fluctuating concentration gradients close to the lateral surfaces of the pipe. Important time-dependent gradients of the in-plane stress normal to the 
fibres appear in those thin regions where prevail the fluctuating or periodic moisture concentrations. Such gradients acting over narrow areas near to the pipe surfaces may create some damages to the structure under hygrothermal fatigue. In the present case, it has been checked that the estimate of the penetration length $e_{0}$ previously proposed by Verchery [4] for plates seems to be satisfactory.

\section{References}

[1] Jacquemin F, Vautrin A. Thick laminated composite pipes submitted to cyclical environmental conditions. In: Proceedings of the 9th European Conference on Composite Materials, ECCM 9, Brighton, 4-7 June, 2000. p. 505-510.
[2] Vautrin A, Jacquemin F. Contribution to the durability analysis of thick composite pipes under hygrothermal fatigue, In: DURACOSYS 2001, 5th International Conference on Durability Analysis of Composite Systems, Tokyo, 6-9 November, 2001.

[3] Jacquemin F. Modelling and simulation of internal stresses in thick cylindrical composite structures, Doctoral dissertation, University Blaise Pascal of Clermont-Ferrand, France, 6 December, 2000 [in French].

[4] Verchery G. Moisture diffusion in polymer matrix composites with cyclical environmental conditions. In: Proceedings of the 5th European Conference on Composite Materials, ECCM 5, Bordeaux, 1992. p. 505-510.

[5] Loos AC, Springer GS. Moisture absorption of graphite-epoxy composition immersed in liquids and in humid air. In: Environmental effects on composite materials. 1981. p. 34-55.

[6] Tsai SW. Composite design, 3rd edition: Think composites. 1987.

[7] Gay D. Composite materials. Paris: Hermès; 1991 [in French]. 